\title{
LETTERS
}

\section{Healthy-vaccinated effect}

In the study by Casado and colleagues, ${ }^{1}$ older inpatients with laboratory-confirmed severe and nonsevere influenza were each matched with three controls who had been hospitalized for causes other than acute respiratory diseases.

The adjusted effectiveness of influenza vaccination in patients who received the vaccine in the current and any previous season, compared with patients who had not been vaccinated in the last four seasons, in preventing hospitalizations was $31 \%$ for nonsevere influenza, $74 \%$ for preventing admission to the intensive care unit and 70\% in preventing death. However, vaccination in the current season only showed no reduced odds of severe disease, and increased deaths (adjusted odds ratio $3.35,1.06-10.58$ ).

The authors conclude that repeated influenza vaccination was twice as effective in preventing severe compared with nonsevere influenza in hospitalized older patients, reinforcing recommendations for annual revaccination.

This conclusion might be misleading, however. Patients with severe influenza had a higher frequency of cardiovascular disease. ${ }^{1}$

A meta-analysis of randomized controlled trials (RCTs) in patients "at high cardiovascular risk" stated that influenza vaccine was associated with a lower risk of major adverse cardiovascular events. ${ }^{2}$ Instead, this advantage was seen only among patients with active coronary artery disease, ${ }^{3}$ without a significant risk reduction in patients with stable coronary artery disease. In patients who did not have the disease, the vaccine showed a tendency to increase the risk of cardiovascular events, cardiovascular mortality and all-cause mortality (relative risk 1.55 [0.96-2.51]). ${ }^{3}$

Influenza vaccination itself induces an inflammatory response, ${ }^{4}$ theoretically increasing the cardiovascular risk, with a number needed to vaccinate to prevent influenza of 40-71 in healthy adults, ${ }^{5}$ higher in older adults. An RCT comparing vaccine versus no intervention in healthy 65- to 74-year-old individuals should address the trade-off, because the "healthy-vaccinated effect" could bias other study designs.

\section{Alberto Donzelli MD}

Specialist in hygiene, public health and preventive medicine, Appropriateness in Primary Care, Health Protection Agency, Metropolitan City of Milan, Italy

\section{Alessandro Schivalocchi MD}

Resident in hygiene, public health and preventive medicine, University of Milan, Bicocca Milano, Italy

\section{Giulia Giudicatti MD}

Resident in hygiene, public health and preventive medicine, University of Milan, Bicocca Milano, Italy

Cite as: CMAJ 2018 July 9;190:E838. doi: 10.1503/cmaj.69406

\section{References}

1. Casado I, Dominguez Á, Toledo D, et al. Project PI12/02079 Working Group. Repeated influenza vaccination for preventing severe and fatal influenza infection in older adults: a multicentre case-control study. CMAJ 2018;190:E3-12.

2. Udell JA, Zawi R, Bhatt DL, et al. Association between influenza vaccination and cardiovascular outcomes in high-risk patients: a meta-analysis. JAMA 2013; 310:1711-20.

3. Donzelli A, Battaggia A. Il vaccino antinfluenzale non sembra apportare benefici in anziani senza malattie cardiovascolari: Revisione critica delle nuove prove da una metanalisi di studi clinici randomizzati controllati. Ric\&Pra 2014;30:149-52 10.1707/1592.17340.

4. Christian LM, Porter K, Karlsson E, et al. Proinflammatory cytokine responses correspond with subjective side effects after influenza virus vaccination. Vaccine 2015;33:3360-6.

5. Demicheli V, Jefferson T, Al-Ansary LA, et al. Vaccines for preventing influenza in healthy adults. Cochrane Database Syst Rev 2014;(3):CD001269.

Competing interests: None declared. 